\title{
Automatic Detection of Texture-defects using Texture-periodicity and Jensen-Shannon Divergence
}

\author{
V. Asha* ${ }^{\star}$ N.U. Bhajantri** and P. Nagabhushan ${ }^{\star \star *}$
}

\begin{abstract}
In this paper, we propose a new machine vision algorithm for automatic defect detection on patterned textures with the help of texture-periodicity and the JensenShannon Divergence, which is a symmetrized and smoothed version of the KullbackLeibler Divergence. Input defective images are split into several blocks of the same size as the size of the periodic unit of the image. Based on histograms of the periodic blocks, Jensen-Shannon Divergence measures are calculated for each periodic block with respect to itself and all other periodic blocks and a dissimilarity matrix is obtained. This dissimilarity matrix is utilized to get a matrix of true-metrics, which is later subjected to Ward's hierarchical clustering to automatically identify defective and defect-free blocks. Results from experiments on real fabric images belonging to 3 major wallpaper groups, namely, pmm, p2, and p4m with defects, show that the proposed method is robust in finding fabric defects with a very high success rates without any human intervention
\end{abstract}

Keywords-Periodicity, Jensen-Shannon Divergence, Cluster, Defect

\section{INTRODUCTION}

Patterned textures are often found in day-to-day life in various applications such as ceramic tiles, wallpapers, and textile fabrics. The inspection of products plays a major role with regard to the quality control of various products in industries. The conventional human-vision based inspection that is being followed in many of the industries has the following shortfalls:

- Lack of repeatability and reproducibility of inspection results because of fatigue and the subjective nature of humans

- Prolonged inspection time

- Imperfect defect detection due to complicated design in textile patterns produced by modern textile industries

Automated machine-vision based inspection will help in reducing the inspection time and in-

Manuscript received September 2, 2011; first revision November 14, 2011; accepted January 2, 2012.

Corresponding Author: V. Asha

* Dept. of Computer Applications, New Horizon College of Engineering, Bangalore, Karnataka - 560103, India \& JSS Research Foundation, SJCE Campus, University of Mysore, Mysore, Karnataka - 570006, India (v_asha@live.com)

** Dept. of Computer Science and Engineering, Govt. Engineering College, Chamarajanagar, Mysore District, Karnataka - 571313, India (bhajan3nu@gmail.com)

*** Dept. of Studies in Computer Science, University of Mysore, Mysore, Karnataka - 570006, India (pnagabhushan@hotmail.com) 
creasing the production rate as compared to the traditional human-vision based inspection. Among the various industries, the textile industry is one of the biggest traditional industries requiring an automated inspection system for increasing production efficiency and reducing the labor costs.

Presently, modern textile industries produce so many varieties in fabric design. Nevertheless, all types of patterned fabrics produced by the modern textile industries can be grouped into only 17 wallpaper groups and are denoted as p1, p2, p3, p3m1, p31m, p4, p4m, p4g, pm, pg, pmg, pgg, p6, p6m, cm, cmm, and pmm [1,2]. These wallpaper groups basically consist of lattices of parallelogram, rectangular, rhombic, square, or hexagonal shapes. A wallpaper group has at least one of the characteristics of translational, rotational, reflectional, and glide-reflectional symmetries. Mathematically, the $\mathrm{p} 1$ wallpaper group is said to be a texture with just one lattice repeating itself over the complete image such as for plain and twill fabrics as shown in Fig. 1(a) and (b). From among the other 16 wallpaper groups pmm, p2, and $\mathrm{p} 4 \mathrm{~m}$ are called major wallpaper groups due to the fact that the wallpaper groups other than pmm, $\mathrm{p} 2$ and $\mathrm{p} 4 \mathrm{~m}$ can be transformed into these 3 major groups via geometric transformation [4]. The shapes of the unit cell in pmm, $\mathrm{p} 2$, and $\mathrm{p} 4 \mathrm{~m}$ wallpaper groups are the rectangle, parallelogram, and square, respectively. Examples of fabrics belonging to these 3 major wallpaper groups are shown in Fig. 1(c)-(e) along with a possible lattice shown superimposed on each major wallpaper group.

Inspection of patterned textures belonging to wallpaper groups other than the $\mathrm{p} 1$ group is more complicated than that in textures belonging to the 1 group due to a complexity in the design, the existence of numerous categories of patterns, and a similarity between the defect and background [3]. As a result, most of the methods in literature depend on the training stage by using numerous defect-free samples for obtaining decision-boundaries or thresholds prior to the detection of defects (i.e., [3-9]). Moreover, methods involving pattern matching and the image subtraction approach perform a pixel-by-pixel comparison, which is inherently sensitive to noises, misalignments, and distortions [8]. Noises, distortions, and misalignments are very common in real fabric images. As a result, all periodic units extracted from a single image cannot have exactly the same intensities. Hence, analyzing periodic units is more appropriate for finding the defects than pixel-by-pixel comparison. Considering all these things, we propose in this paper a method of defect detection for fabric images belonging to 16 out of 17 wallpaper groups without any training stage via the help of texture-periodicity and the Jensen-Shannon Divergence. This will increase the production efficiency by decreasing the inspection time. The main contributions of this research can be summarized as follows:

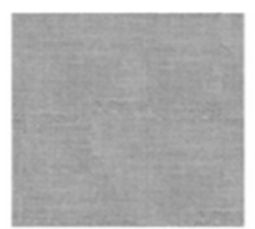

(a)

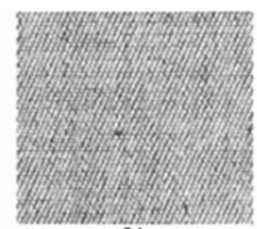

(b)

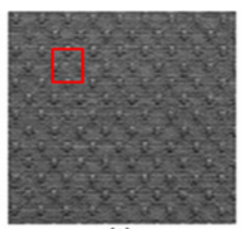

(c)

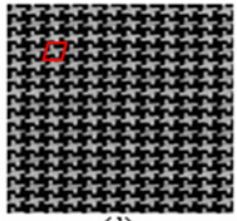

(d)

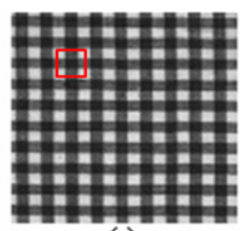

(e)

Fig. 1. Examples of textile fabrics: (a) Plain fabric (p1 group); (b) Twill fabric (p1 group); (c) Dotpatterned fabric (pmm group); (d) Star-patterned fabric (p2 group); (e) Box-patterned fabric (p4m group). Lattice shapes of the three major wallpaper groups (pmm, p2 and pm) are rectangle, parallelogram and square. A possible lattice is shown superimposed on each major wallpaper group 
- The proposed method of defect detection is more generic as the method can be applied to periodic images belonging to 16 out of 17 wallpaper groups (other than the 1 group for images such as plain and twill fabric images).

- The proposed method does not require any training stage with defect-free samples for creating decision boundaries or thresholds unlike with other methods. As a result, the proposed method does not need a huge memory space for the storage of defect-free samples.

- Detection of defective and defect-free periodic units is automatically carried out based on cluster analysis without human intervention.

The program for the proposed algorithm is written in Matlab-7.0 and is run on a Pentium-IV personal computer with a RAM capacity of 2 GB. The organization of this paper is as follows: Section 2 presents a brief review on the different methods available in literature for defect detection of patterned textures. Section 3 presents a brief review on the Jensen-Shannon Divergence and the proposed algorithm for defect detection along with illustrations and results from experiments on various real fabric images with defects. Section 4 has the conclusions.

\section{Previous Methods}

Methods that are found in literature for the inspection of patterned texture images include the traditional image subtraction methods [10-12], the method of golden image subtraction (GIS) [5], the method of wavelet-preprocessed golden image subtraction (WGIS) [5], the method of Direct-Thresholding (DT) based on wavelet transform [5], the Bollinger Bands method [6], the Regular Bands method [3], the Local Binary Pattern (LBP) method [7], and the motif-based methods $[4,8,9]$.

The traditional image subtraction method developed by Chin and Harlow for the inspection of printed circuit boards involves a direct subtraction of the image that is under inspection with a defect-free template image [10]. Since this method involves pixel to pixel comparison, it is sensitive to noises and distortions. Khalaj et al. developed a method of inspecting patterned wafers based on periodicity estimation using a gray value projection and a reference image that is constructed from the input image itself using the average gray values of all the periodic units [11]. Pixel-to-pixel comparison between the input image and the reference or template image, which is based on an assumed threshold, helps in identifying the defects. Xie and Guan presented a similar method, wherein the building block needed for constructing a reference image is extracted based on linear interpolation [12]. However, when the defect size in the image is too large, the building block constructed based on the methods suggested in $[11,12]$ can never be a good estimate of the true value.

The basic GIS method involves a training stage with lot of defect-free samples and a testing stage [5]. In the training stage, the energy of the golden image subtraction, which is defined as the sum of absolute difference between the golden image (a template unit of size that is more than that of the periodic unit) and a histogram-equalized reference image (defect-free image) over a given window, is obtained at every pixel location. Thresholds are obtained from several defect-free samples. In the testing stage, energies obtained from the golden image and the defective test images are compared with the thresholds obtained from the training stage to find the defects after using a median filter or Weiner filter to perform filtering. The method was tested 
with 30 defect-free and 30 defective pmm images. The detection success rates obtained for the pmm images are $100 \%$ for defect-free images and $56.67 \%$ for defective images. The overall success rate was found to be $78.33 \%$. In order to overcome the sensitivity of this method to noise, the WGIS method was developed [5]. This is similar to the GIS method expect that a Haar wavelet transform is applied over all the images and the sub-images (in level-1 approximation) are utilized instead of the original image. The overall success rate was improved to $96.7 \%$. In the method of DT [5], the Haar wavelet transform is applied to the reference images and the fourth level horizontal and vertical details are extracted. Lower and upper bound values of the three horizontal details in level-4 and also vertical details are extracted and their averages are calculated after filtering. Thresholds obtained using these horizontal and vertical details in the training stage with defect-free images are utilized in the testing stage for finding the defects in pmm images. The detection success rates were found to be $86.77 \%$ for defect-free images and $90 \%$ for defective images. The overall detection success rate was found to be $88.3 \%$.

The Bollinger Bands are basically measures of oversold and overbought trends in stock markets, and are calculated based on the moving average and standard deviation. Ngan and Pang extended Bollinger Bands (BB) from a one-dimensional approach into a two-dimensional approach for defect detection on patterned Jacquard fabrics belonging to the pmm group [6]. The application of $\mathrm{BB}$ for defect detection is in two stages, namely, the training stage and testing stage. Upper and lower Bollinger bands are calculated for the reference images in the training stage based on the mean and standard deviations and the size of the repetitive unit and thresholds are obtained. The thresholds are later used for detecting defects from defective images in the testing stage. The method was tested on the pmm, p2, and p4m wallpaper groups with 167 defect-free images and 171 defective images and the detection success rate was found to be $98.59 \%$.

Ngan and Pang presented another approach called the Regular Bands (RB) method for defect detection on patterned textures. This was done with the help of the moving average and standard deviation [3]. Thresholds obtained from several histogram-equalized defect-free images during the training stage were used for detecting defects on histogram-equalized defective images during the testing stage. The method was tested on the pmm, p2, and $\mathrm{p} 4 \mathrm{~m}$ wallpaper groups with 80 defect-free images and 86 defective images and the detection success rate was found to be 99.4\%.

Fabric defect detection using the modified local binary pattern (LBP) [7] involves two stages, namely, the training stage and the defect detection stage. In the training stage, the LBP operator is applied to an image of defect-free fabric pixel-by-pixel, and a reference feature vector is computed. The defect-free fabric is then divided into several windows of size that are slightly more than that of periodic unit and an LBP operator is applied to each of these windows to get a suitable threshold from the defect-free image. In the detection stage the defective fabric is divided into several windows (as in the training stage) and LBPs are obtained. Defects are then located in the fabric based on the threshold. The method was tested on pmm, p2, and $\mathrm{p} 4 \mathrm{~m}$ images and the detection success rate was found to be $96.7 \%$.

Ngan et al. [4, 8, 9] developed motif-based methods for detecting defective lattices from 16 out of 17 wallpaper groups based on energy and the variance of the hand-located lattices. Minimum-maximum decision boundaries (rectangular boundaries) are obtained in an energyvariance space from several defect-free test images using hand-located defect-free and defective lattices that are said to be composed of motifs $[4,8]$. The energy of the moving subtraction be- 
tween a motif and its circular shift matrices is derived using a norm-metric measurement and the variance of the energies for all motifs is obtained. By learning the distribution of these values over a number of defect-free lattices, boundary conditions for discerning defective and defectfree lattices are obtained. As the 16 wallpaper groups of patterned fabric can be transformed into three major groups, namely, pmm, p2, and p4m, the method was evaluated over these three major wallpaper groups. Decision boundaries were obtained using 160 defect-free lattices samples and the method was tested with 140 defect-free and 113 defective samples. An overall detection success rate of $93.3 \%$ was achieved. Ngan et al. [9] later proved that instead of minimummaximum boundaries, when ellipsoidal decision boundaries are utilized (based on the assumption that energy-variance values of defect-free lattices follow a Gaussian distribution), the average detection success rate increases to $95.8 \%$.

\section{Proposed Algorithm for Defect Detection}

The basic idea for the proposed algorithm for defect detection is to divide the input texture image into several periodic blocks and analyze those blocks for defects. The Jensen-Shannon Divergence is one of the effective measures for capturing mutual information between two probability distributions [13]. In this paper, we intend to employ the Jensen-Shannon Divergence metric to discriminate between defective and defect-free periodic blocks.

\subsection{Brief Review on the Jensen-Shannon Divergence}

The Jensen-Shannon Divergence is a symmetrized and smoothed out version of the KullbackLeibler Divergence, which is the most important divergence measure of information theory [13]. Given two classes $\omega_{1}$ and $\omega_{2}$ with a common feature vector $\boldsymbol{x}$, the Kullback-Leibler Divergence or the relative entropy in terms of the ratio of the probability distributions $p\left(\omega_{1}\right)$ and $p\left(\omega_{2}\right)$ of these classes conveys useful information concerning the capability of discriminating between the two classes. This class separability measure over class $\omega_{1}$ is given as [14]:

$$
\lambda\left(\omega_{1}, \omega_{2}\right)=\int_{-\infty}^{\infty} p\left(\omega_{1}\right) \log \left(\frac{p\left(\omega_{1}\right)}{p\left(\omega_{2}\right)}\right) d x
$$

Similarly, the class separability measure over class $\omega_{2}$ is given as:

$$
\lambda\left(\omega_{2}, \omega_{1}\right)=\int_{-\infty}^{\infty} p\left(\omega_{2}\right) \log \left(\frac{p\left(\omega_{2}\right)}{p\left(\omega_{1}\right)}\right) d x
$$

Based on the probability distributions $p\left(\omega_{1}\right)$ and $p\left(\omega_{2}\right)$ and their average, a symmetric divergence called the Jensen-Shannon Divergence $(\Lambda)$ is given as:

$$
\Lambda=\int_{-\infty}^{\infty}\left\{p\left(\omega_{1}\right) \log \left(\frac{0.5 \times p\left(\omega_{1}\right)}{p\left(\omega_{1}\right)+p\left(\omega_{2}\right)}\right)+p\left(\omega_{2}\right) \log \left(\frac{0.5 \times p\left(\omega_{2}\right)}{p\left(\omega_{1}\right)+p\left(\omega_{2}\right)}\right)\right\} d x
$$


Since the Kullback-Leibler Divergence $\lambda\left(\omega_{1}, \omega_{2},\right)$ or $\lambda\left(\omega_{2}, \omega_{1}\right)$ can be interpreted as the inefficiency of assuming that the true distribution is $p\left(\omega_{2}\right)$ or $p\left(\omega_{1}\right)$ when it is really $p\left(\omega_{1}\right)$ or $p\left(\omega_{2}\right)$, the Jensen-Shannon Divergence could be considered as a minimum inefficiency distance [13]. The square-root of this divergence is a true metric that obeys the following properties of a true metric in a 2D Euclidean-space:

(i) Non-negativity $\left(\Lambda_{i j}>0\right)$, for all $i$ and $j$

(ii) Self-distance $\left(\Lambda_{i j}=0\right)$, if $i=j$

(iii) Symmetry $\left(\Lambda_{i j}=\Lambda_{j i}\right)$ for all $i$ and $j$

(iv) Triangular-inequality $\left(\sqrt{\Lambda_{i k}} \leq \sqrt{\Lambda_{i j}+\Lambda_{j k}}\right)$ for $i \neq j \neq k$

\subsection{Description of the Algorithm}

For non-periodic applications, there is no restriction on the size of the window to be used for analyzing the image for defects. In the case of periodically patterned textures, the size of the window to be used for defect detection can be chosen to be the size of the periodic unit of the image itself instead of selecting the window size to be more than the size of the periodic unit or less than the size of the periodic unit. The proposed algorithm specifically aims towards defect detection on periodically patterned textures under the following assumptions:

- The test image is of at least two periodic units in a horizontal direction and two in a vertical direction whose dimensions are known a priori.

- The number of defective periodic units is always less than the number of defect-free periodic units.

- Test images are from the imaging system that is oriented perpendicular to the surface of the product such as a textile fabric. This assumption is due to the fact that in a defect detection system in industries such as the fabric industry, the imaging system is always oriented perpendicular to the plane of the fabric surface.

Because the main idea of the proposed method is to analyze the periodic blocks from the test image, four cropped images are obtained from the defective test image by cropping the input image from all four corners (top-left, bottom-left, top-right, and bottom-right) to get the complete number of periodic blocks based on [15]. If $g$ is an image of size $M \times N$ with row periodicity $P_{r}$ (i.e., the number of columns in a periodic unit) and column periodicity $P_{c}$ (i.e., number of rows in a periodic unit), then the size of the cropped image $g_{\text {crop }}$ is $M_{\text {crop }} \times N_{\text {crop }}$ where $M_{\text {crop }}$ and $N_{\text {crop }}$ are measured from the top-left, bottom-left, top-right, and bottom-right corners and are given by the following equations using floor operation in mathematics:

$$
\begin{gathered}
M_{\text {crop }}=\left\lfloor M / P_{c}\right\rfloor \times P_{c} \\
N_{\text {crop }}=\left\lfloor N / P_{r}\right\rfloor \times P_{r}
\end{gathered}
$$

Each cropped image is split into several periodic blocks of size $P_{c} \times P_{r}$. If $p$ and $q$ represent the probability distributions of two periodic blocks $A$ and $B$ whose dynamic range of gray values 
is $[0, L-1]$, the Jensen-Shannon Divergence can be computed as [13]:

$$
\Lambda_{A, B}=\Lambda_{B, A}=\sum_{k=0}^{L-1} p\left(r_{k}\right) \log \left(\frac{0.5 \times p\left(r_{k}\right)}{p\left(r_{k}\right)+q\left(r_{k}\right)}\right)+\sum_{k=0}^{L-1} q\left(r_{k}\right) \log \left(\frac{0.5 \times q\left(r_{k}\right)}{p\left(r_{k}\right)+q\left(r_{k}\right)}\right)
$$

where the probability distributions of the two periodic blocks $A$ and $B$ are given by:

$$
p\left(r_{k}\right)=\frac{n_{A, i}}{n}, q\left(r_{k}\right)=\frac{n_{B, i}}{n}, \mathrm{i}=0,1,2, \ldots, \mathrm{L}-1
$$

where $n_{A, i}$ is the number of pixels that have gray level $r_{k}$ in the image $A, n_{B, i}$ is the number of pixels that have gray level $r_{k}$ in the image $B, n$ is the total number of pixels in the image $A$ or $B$, and $L$ is the total number of gray values in the periodic block $A$ or $B$. Following (6), the JensenShannon Divergence metric measures are calculated for each periodic block with respect to itself and all other periodic blocks to get a dissimilarity matrix $\boldsymbol{D}$ as below:

$$
D=\left[\begin{array}{ccccc}
\sqrt{\Lambda_{1,1}} & \sqrt{\Lambda_{1,2}} & \cdots & \sqrt{\Lambda_{1, n-1}} & \sqrt{\Lambda_{1, n}} \\
\sqrt{\Lambda_{2,1}} & \sqrt{\Lambda_{2,2}} & \cdots & \sqrt{\Lambda_{2, n-1}} & \sqrt{\Lambda_{2, n}} \\
\vdots & \vdots & \vdots & \vdots & \vdots \\
\sqrt{\Lambda_{n-1,1}} & \sqrt{\Lambda_{n-1,2}} & \cdots & \sqrt{\Lambda_{n-1, n-1}} & \sqrt{\Lambda_{n-1, n}} \\
\sqrt{\Lambda_{n, 1}} & \sqrt{\Lambda_{n, 2}} & \cdots & \sqrt{\Lambda_{n, n-1}} & \sqrt{\Lambda_{n, n}}
\end{array}\right]
$$

It should be noted that if a cropped image has $n$ number of periodic blocks, then the size of the dissimilarity matrix is $n \times n$. Because the divergence measure of a periodic block with itself is zero and the divergence measure between the $i^{\text {th }}$ periodic block and the $j^{\text {th }}$ periodic block is same as the divergence measure between the $j^{\text {th }}$ periodic block and the $i^{\text {th }}$ periodic block, the dissimilarity matrix becomes a hollow matrix (diagonally symmetric matrix with diagonal elements being zero) as below:

$$
D=\left[\begin{array}{ccccc}
0 & & & & \\
\sqrt{\Lambda_{2,1}} & 0 & & & \\
\vdots & \vdots & \ddots & & \\
\sqrt{\Lambda_{n-1,1}} & \sqrt{\Lambda_{n-1,2}} & \cdots & 0 & \\
\sqrt{\Lambda_{n, 1}} & \sqrt{\Lambda_{n, 2}} & \cdots & \sqrt{\Lambda_{n, n-1}} & 0
\end{array}\right]
$$

It may be noted that the upper diagonal elements are not filled for the sake of simplicity. In order to extract defective and defect-free clusters from this dissimilarity matrix, Ward's hierarchical algorithm, which is based on inner squared distance and minimum variance criterion [14], is utilized and a cluster tree is obtained in the form of the linkage matrix $Z$ of size $(n-1) \times 3$. The leaf nodes in the cluster hierarchy are the periodic blocks in the original data set numbered from 1 to $n$. These are the singleton clusters from which all higher clusters are built. Each newly formed cluster, corresponding to row $i$ in $Z$, is assigned the index $n+i$. The first and second 
columns of the linkage matrix contain the indices of the periodic blocks that were linked in pairs to form a new cluster. This new cluster is assigned the index value $n+i$. There are $n-1$ higher clusters corresponding to the interior nodes of the hierarchical cluster tree. The third column contains the corresponding linkage distances between the periodic blocks paired in the clusters at each row $i$. The last value of the linkage distance is maximum, which indicates that all periodic blocks are grouped into one cluster and the last but one value of the linkage distance is the next maximum corresponding to two clusters yielding the required cut-off point at which two clusters (one cluster containing defective periodic blocks and the other cluster containing defectfree periodic blocks) are formed. Upon identifying the two clusters, the number of periodic blocks in one cluster is compared with that of the other and the cluster with the less number of periodic blocks is assumed to be defective. Detection of defective periodic blocks from each cropped image does not give an overview of the total defects in the input defective image. Hence, in order to get the overview of the total defects in the input image, we use defect-fusion, which is proposed in [15]. The boundaries of the defective periodic blocks identified from each cropped image are merged together and morphologically filled [16] to get fused defective zones. Edges of these fused defects are extracted using the Canny edge operator [16] and are superimposed on the original defective image to get the overview of the total defects in the input image. The entire procedure for defect detection is shown in the form of a flow chart in Fig. 2. Summarizing the entire procedure for defect detection, the pseudo-codes for the algorithm can be given using the following steps:

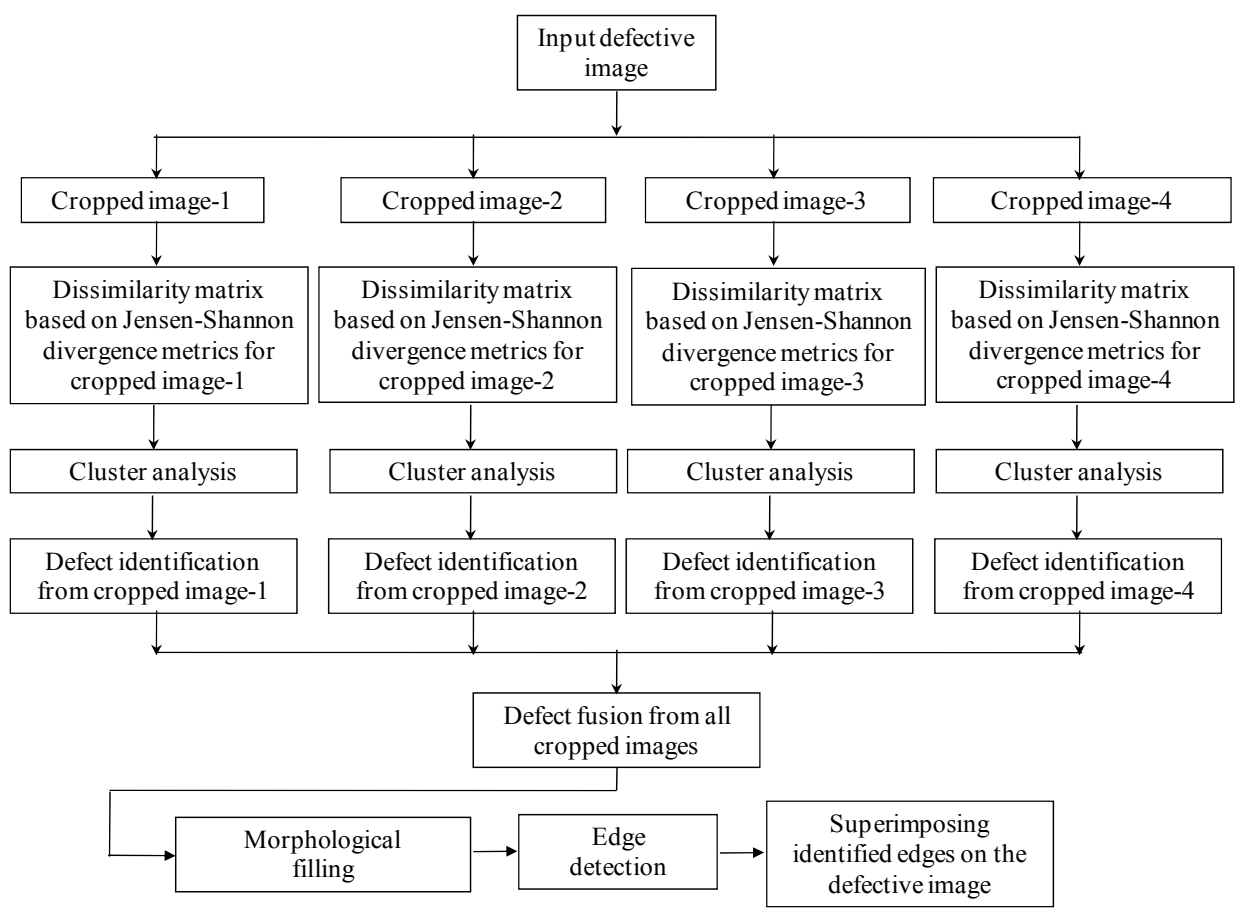

Fig. 2. Proposed defect detection scheme 
(i) Obtain four cropped images from the input defective image based on equations (4) and (5) using periodicities.

(ii) Split each cropped image into several periodic blocks and obtain a histogram of each periodic block.

(iii) Calculate the Jensen-Shannon Divergences of each periodic block with respect to itself and all other periodic blocks following equation (6) for each cropped image.

(iv) Formulate a dissimilarity matrix containing the Jensen-Shannon Divergence metrics as shown in equation (8) for each cropped image.

(v) Perform Ward's hierarchical clustering on the dissimilarity matrix and identify the defective periodic blocks from each cropped image.

(vi) Fuse the boundaries of the defective blocks identified from all four cropped images and perform morphological filling.

(vii) Extract the edges of the morphologically filled zones and superimpose the extracted edges on the original defective image to get an overview of the total defects.

\subsection{Illustration of the Algorithm}

In order to illustrate the proposed algorithm for defect detection, let us look at a defective box-patterned fabric image (p4m group) as shown in Fig. 3(a).

Following (4) and (5), four cropped images containing a complete number of periodic blocks are obtained, as shown in Fig. 3(b)-(e), from the test image with the help of periodicities known a priori. Each cropped image is split into several blocks that are of the same size as the size of the periodic unit as shown in Fig. 4(a) for a sample cropped image and each periodic block is numbered as shown in Fig. 4(b).

The Jensen-Shannon Divergence metrics are calculated for each periodic block with respect to itself and all other periodic blocks and a dissimilarity matrix is obtained using (8). The dissimilarity matrices thus obtained are shown in Fig. 5 in gray-scale form by scaling the matrix elements linearly in the range $0-255$. The intensity value " 0 " in the dissimilarity matrix at any location $(i, j)$ indicates that the periodic blocks $i$ and $j$ are identical. The intensity value "255" in the dissimilarity matrix at $(i, j)$ indicates that the periodic blocks $i$ and $j$ are of maximum dissimilarity with each other. It is clear from Fig. 5 that the diagonal elements in the dissimilarity matrix are " 0 ," which implies that the periodic blocks are of zero dissimilarity with themselves and that

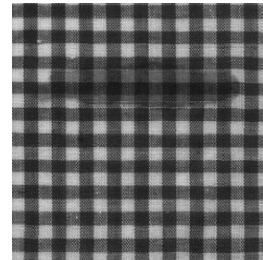

(a)

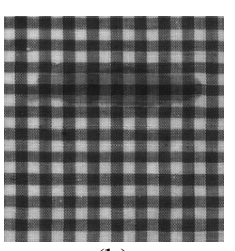

(b)

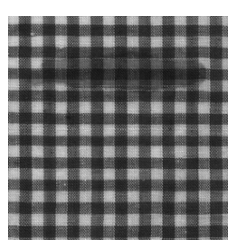

(c)

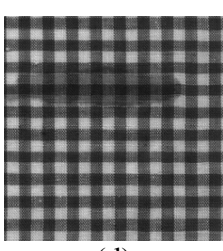

(d)

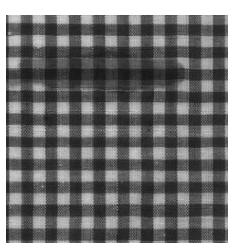

(e)

Fig. 3. (a) Input defective image; (b) Cropped image obtained from top-left corner of the input image; (c) Cropped image obtained from bottom-left corner of the input image; (d) Cropped image obtained from top-right corner of the input image; (e) Cropped image obtained from bottom-right corner of the input image 


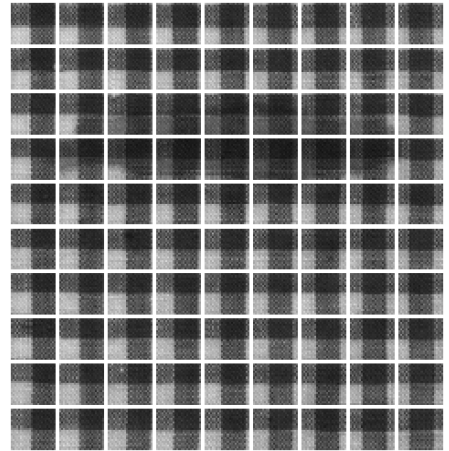

(a)

\begin{tabular}{|r|l|l|l|l|l|l|l|l|}
\hline 1 & 11 & 21 & 31 & 41 & 51 & 61 & 71 & 81 \\
\hline 2 & 12 & 22 & 32 & 42 & 52 & 62 & 72 & 82 \\
\hline 3 & 13 & 23 & 33 & 43 & 53 & 63 & 73 & 83 \\
\hline 4 & 14 & 24 & 34 & 44 & 54 & 64 & 74 & 84 \\
\hline 5 & 15 & 25 & 35 & 45 & 55 & 65 & 75 & 85 \\
\hline 6 & 16 & 26 & 36 & 46 & 56 & 66 & 76 & 86 \\
\hline 7 & 17 & 27 & 37 & 47 & 57 & 67 & 77 & 87 \\
\hline 8 & 18 & 28 & 38 & 48 & 58 & 68 & 78 & 88 \\
\hline 9 & 19 & 29 & 39 & 49 & 59 & 69 & 79 & 89 \\
\hline 10 & 20 & 30 & 40 & 50 & 60 & 70 & 80 & 90 \\
\hline
\end{tabular}

(b)

Fig. 4. (a) Sample cropped image split into several periodic blocks; (b) Numbering sequence followed for the periodic blocks

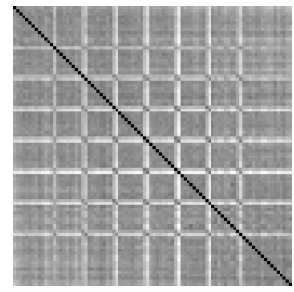

(a)

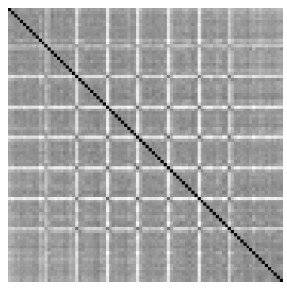

(b)

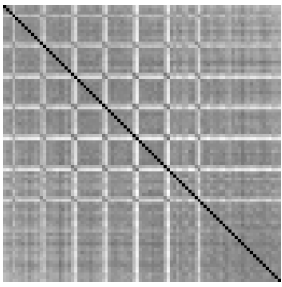

(c)

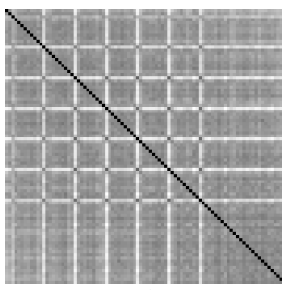

(d)

Fig. 5. Dissimilarity matrix derived from the Jensen-Shannon divergence measures for the cropped image obtained from (a) top-left (b) bottom-left (c) top-right and (d) bottom-right corners of the test image shown in gray-scale

the dissimilarity matrix is symmetric. The dendrograms obtained from the dissimilarity matrices through clustering are shown in Fig. 6 along with the defective periodic blocks identified by the clustering for all cropped images. Boundaries of the defective periodic blocks thus identified from each cropped image are highlighted using white pixels and are shown in Fig. 7. The boundaries of the defective periodic blocks identified from each cropped image are shown superimposed on the original image in Fig. 8(a) and separately on a plain background in Fig. 8(b). Fig. 8(c) shows the result of morphological filling and Fig. 8(d) shows the edges of the total defects superimposed on the original defective image after Canny edge detection. Thus, we can get an overview of the total defects on the original image itself following the concept of fusion, morphological filling, and edge detection.

It may be noted that though the number of periodic blocks taken from a defective input image is the same for all of its cropped images, the number of defective periodic blocks identified does not need to be the same for all cropped images. This is because the contribution of defects in each periodic block may differ for different cropped images. Nevertheless, the fusion of defects from all 4 cropped images helps in getting an overview of the total defects in the input image. This is clearly evident from the example image used for illustration of the proposed algorithm. 


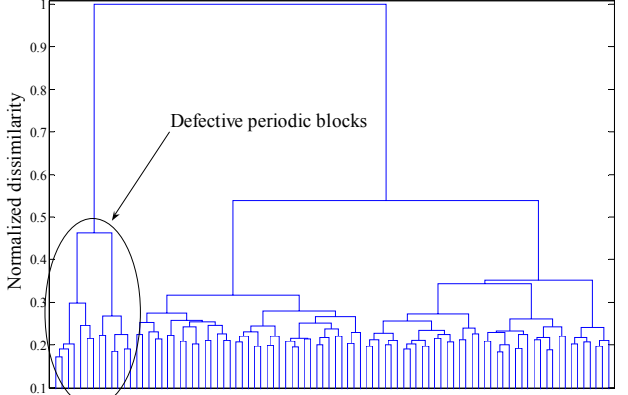

Periodic block identity

(a)

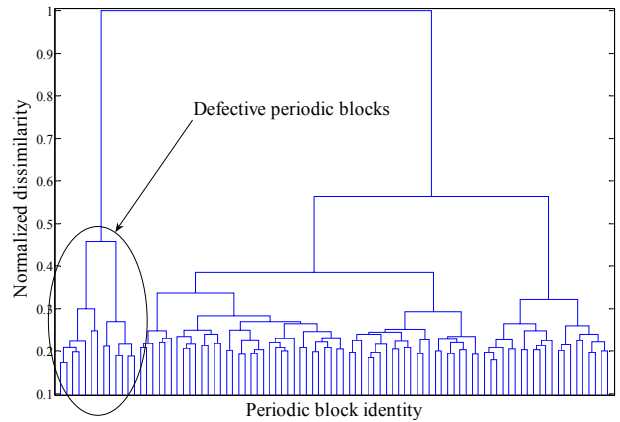

(c)

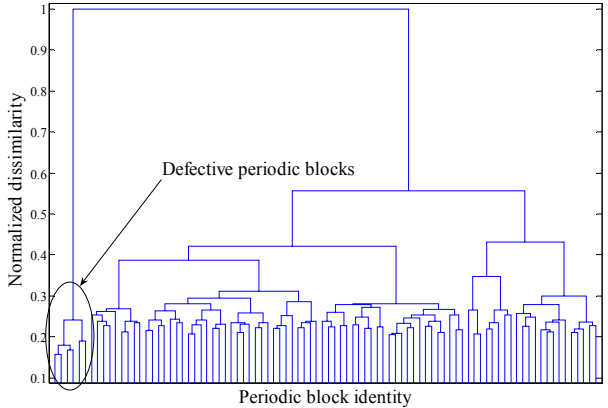

(b)

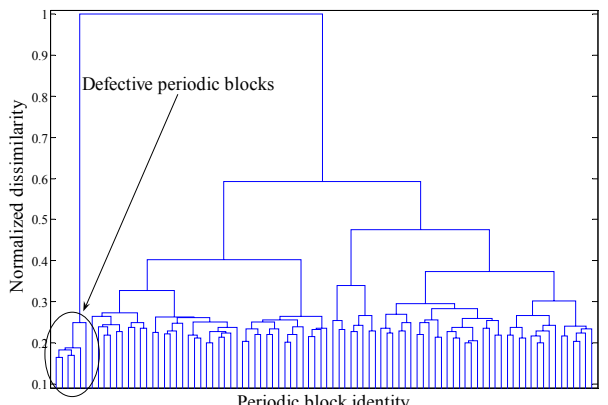

(d)

Fig. 6. Dendrograms obtained from cluster analysis of the Jensen-Shannon divergence matrix obtained from the test image by cropping it from (a) top-left (b) bottom-left (c) top-right and (d) bottom-right corners. Defective blocks identified from these cropped images are $(14,24$, $74,34,44,64,54,23,73,33,53,43$, and 63$),(43,63,33,53,23$, and 73$),(4,14,24,34$, $44,54,64,13,63,23,53,33$, and 43$)$ and $(33,53,23,43,63$, and 13$)$. It may be noted that since the cropped images have more number of periodic blocks, the identities of the periodic blocks in the abscissa are not shown in order to avoid crowd and to have better clarity

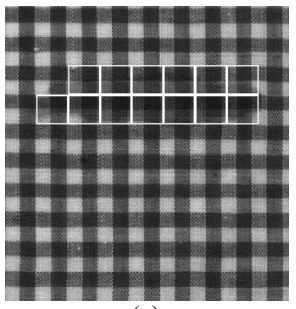

(a)

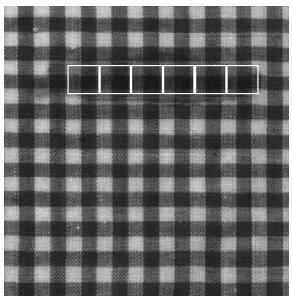

(b)

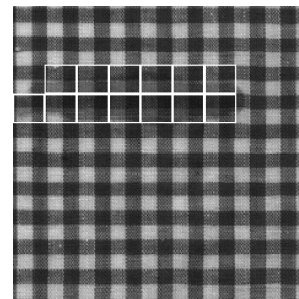

(c)

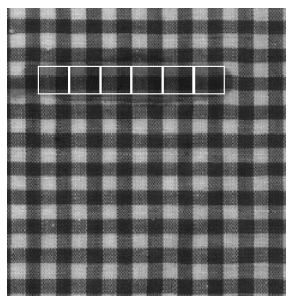

(d)

Fig. 7. Defective periodic blocks identified from the cluster analysis of dissimilarity matrix derived from the Jensen-Shannon divergence measure of the cropped images obtained from (a) top-left (b) bottom-left (c) top-right and (d) bottom-right corners of the test image with their boundaries highlighted using white pixels 


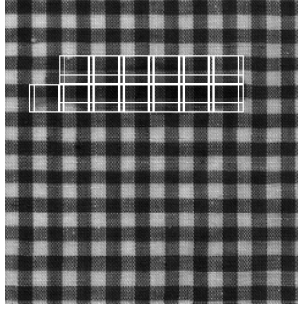

(a)

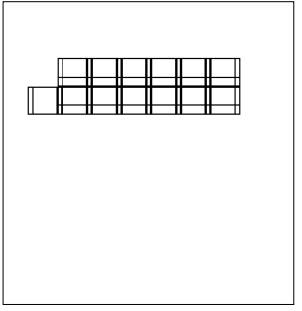

(b)

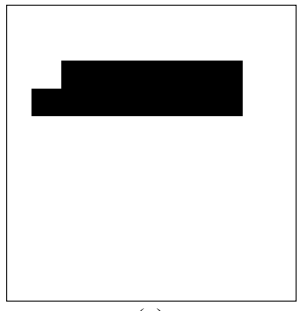

(c)

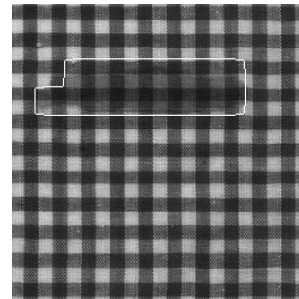

(d)

Fig. 8. Illustration of defect fusion: (a) Boundaries of the defective blocks identified from each cropped image shown superimposed on the original image; (b) Boundaries of the defective blocks shown separately on plain background; (c) Result of morphological filling; (d) Edges identified through Canny edge operator shown superimposed on original defective image using white pixels

\subsection{Experiments on Fabric Images of 3 Major Wallpaper Groups}

In order to study the performance of the proposed algorithm for different wallpaper groups, fabric images belonging to three major wallpaper groups (the pmm, p2, and $\mathrm{p} 4 \mathrm{~m}$ groups whose lattices are of rectangular, parallelogram, and square shapes, respectively) with defects such as a broken end (BE), thin bar (TNB), and thick bar (TKB) are tested. As far as defect detection based on lattice concept is concerned, the reason behind choosing the pmm, p2, and p4m wallpaper groups is that all other wallpaper groups can be transformed into these 3 wallpaper groups through geometric transformations [4]. However, analyzing a wallpaper group (with square or rectangular lattices) with orthogonal periodicities is easier than analyzing a wallpaper group (with parallelogram or rhombus or hexagon lattices) with non-orthogonal periodicities. Analyzing the wallpaper group with square or rectangular lattices involves only orthogonal periodicities (i.e., row and column periodicities). An image can be easily split into several rectangular or square periodic blocks and each periodic block can be analyzed for finding the defect. Hence, the proposed method is aimed at defect detection on the basis of orthogonal periodicities (horizontal and vertical periodicities) instead of non-orthogonal periodicities. Also, there is no need for the geometric transformation of wallpaper groups other than transforming pmm, $\mathrm{p} 2$, and $\mathrm{p} 4 \mathrm{~m}$ into $\mathrm{pmm}, \mathrm{p} 2$, and $\mathrm{p} 4 \mathrm{~m}$ groups. The average sizes of the periodic units for $\mathrm{pmm}$, $\mathrm{p} 2$, and $\mathrm{p} 4 \mathrm{~m}$ test images shown in Fig. 9 are $36 \times 26$ pixels, $16 \times 21$ pixels, and $24 \times 26$ pixels, respectively, and are known as a priori. The final result of defect detection after fusion, morphological filling, and edge detection are shown in Fig. 9 for the defective fabric images of the pmm, p2, and p4m wallpaper groups. 


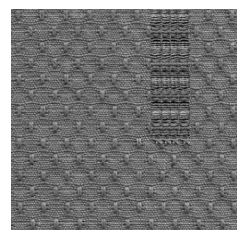

(a)

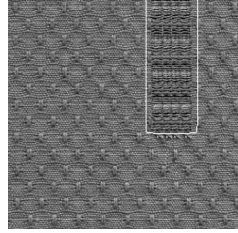

(d)

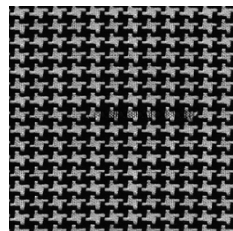

(g)

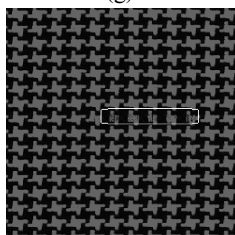

(j)

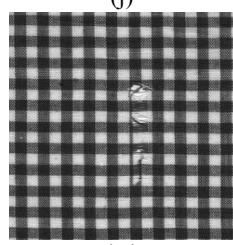

(m)

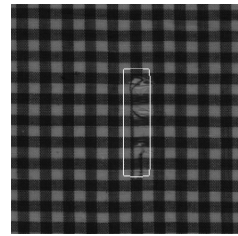

(p)

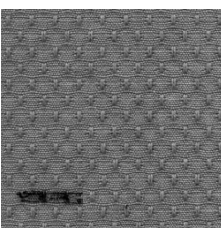

(b)

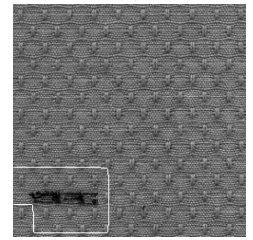

(e)

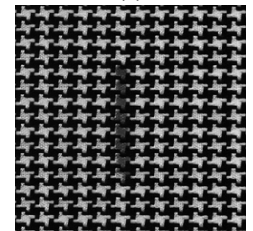

(h)

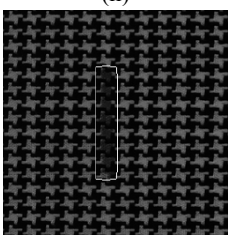

(k)

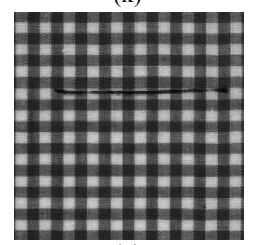

(n)

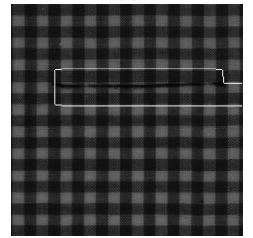

(q)

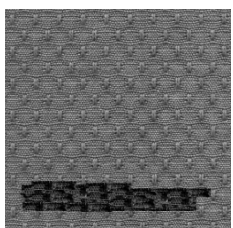

(c)

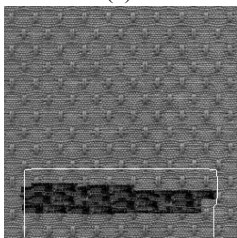

(f)

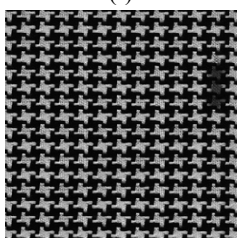

(i)

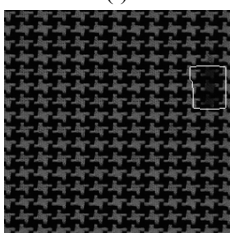

(1)

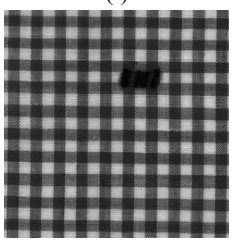

(o)

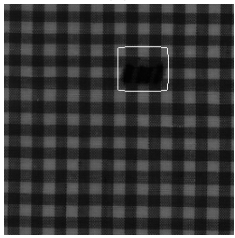

(r)

Fig. 9. Final result of defect detection on 3 major wallpaper groups after defect fusion, morphological filling and edge detection: (a), (b), and (c) represent pmm images with defects - broken end, thin bar and thick bar; (d), (e) and (f) show the final result of defect detection from the defective pmm images; $(\mathrm{g})$, (h), and (i) represent $\mathrm{p} 2$ images with defects - broken end, thin bar and thick bar; (j), (k) and (l) show the final result of defect detection from the defective $\mathrm{p} 2$ images; $(\mathrm{m}),(\mathrm{n})$, and (o) represent p4m images with defects - broken end, thin bar and thick bar; $(p),(q)$ and $(r)$ show the final result of defect detection from the defective $\mathrm{p} 4 \mathrm{~m}$ images. It may be noted that since the star patterns in $\mathrm{p} 2$ images are too bright, gray values in all p2 images in the range $0-255$ are linearly scaled down to $0-127$ so as to make the boundaries of the defects appear with better clarity to viewers 


\subsection{Performance Evaluation of the Proposed Defect Detection Algorithm}

In order to access the performance of the proposed method, performance parameters $[17,18]$, namely, precision, recall, and accuracy are all calculated in terms of true positive (TP), true negative (TN), false positive (FP), and false negative (FN). True positive is defined as the number of defective periodic blocks identified as defective, true negative is defined as the number of defect-free periodic blocks identified as defect-free, false positive is defined as the number of defect-free periodic blocks identified as defective, and false negative is defined as the number of defective periodic blocks identified as defect-free. Precision is defined as the number of periodic blocks correctly labeled as belonging to the positive class divided by the total number of periodic blocks that are labeled as belonging to the positive class and is calculated as TP/(TP+FP). Recall is defined as the number of true positives divided by the sum of true positives and false negatives that are periodic blocks that are not labeled as belonging to the positive class but should have been and it is calculated as $\mathrm{TP} /(\mathrm{TP}+\mathrm{FN})$. Accuracy is the measure of success rate that considers the detection rates of defective and defect-free periodic blocks and is calculated as $(\mathrm{TP}+\mathrm{TN}) /(\mathrm{TP}+\mathrm{TN}+\mathrm{FP}+\mathrm{FN})$. The performance parameters averaged over all the cropped images for each defective image are given in Table 1 for the pmm, $\mathrm{p} 2$, and $\mathrm{p} 4 \mathrm{~m}$ groups. The number of periodic blocks in the pmm group is 63 per cropped image. The number of periodic blocks in the p2 group is 165 per cropped image. The number of periodic blocks in the $\mathrm{p} 4 \mathrm{~m}$ group is 90 per cropped image. The performance parameters are again averaged (based on the total number of periodic samples -- 756, 990, and 1,080 for the pmm, p2, and p4m wallpaper groups, respectively) for all the defective images and are given in Table 2 . The average success rates for the 3 major wallpaper groups in the proposed method of defect detection (without any training stage) are $97.9 \%, 99.3 \%$, and $99.3 \%$ respectively. The average success rate obtained in our method for all three groups is $98.8 \%$, which is on par with the results of methods involving the training stage with numerous defect-free samples, namely, the Bollinger Bands method [6], the Regular Bands method [4], the LBP-based method [7], the motif-based method with minimummaximum decision boundaries [8], and the motif-based method with ellipsoidal decision boundaries [9], which are $98.59 \%, 99.4 \%, 96.7 \%, 93.3 \%$, and $95.8 \%$, respectively. It may be noted that relatively less recall rates in the proposed method indicate that there are few false negatives identified by the proposed method. However, since the proposed method yields very

Table 1. Summary of the performance parameters for each defective image (Note: BE=broken end, $\mathrm{TNB}=$ thin bar, and TKB=thick bar)

\begin{tabular}{c|c|c|c|c|c}
\hline Wallpaper group & Defect & No. of periodic blocks & Precision (\%) & Recall (\%) & Accuracy (\%) \\
\hline \multirow{4}{*}{ pmm } & BE & 252 & 100 & 80.0 & 96.8 \\
\cline { 2 - 6 } & TNB & 252 & 100 & 87.5 & 99.2 \\
\cline { 2 - 6 } & TKB & 252 & 100 & 91.7 & 97.6 \\
\hline \multirow{4}{*}{ p2 } & BE & 330 & 100 & 81.7 & 99.4 \\
\cline { 2 - 6 } & TNB & 330 & 100 & 100 & 100 \\
\cline { 2 - 6 } & TKB & 330 & 100 & 58.3 & 98.5 \\
\hline \multirow{3}{*}{$p 4 m$} & BE & 360 & 100 & 91.7 & 99.4 \\
\cline { 2 - 6 } & TNB & 360 & 100 & 93.8 & 99.4 \\
\cline { 2 - 6 } & TKB & 360 & 100 & 62.5 & 98.9 \\
\hline
\end{tabular}


Table 2. Summary of the performance parameters that have been averaged for all defective images

\begin{tabular}{c|c|c|c}
\hline Wallpaper group & Precision (\%) & Recall (\%) & Accuracy (\%) \\
\hline pmm & 100 & 86.4 & 97.9 \\
\hline p2 & 100 & 80.0 & 99.3 \\
\hline p4m & 100 & 82.6 & 99.3 \\
\hline Average & 100 & 83.0 & 98.8 \\
\hline
\end{tabular}

high precision and accuracy, the proposed method can contribute to automatic defect detection in fabric industries.

\section{CONCLUSION}

In this paper, texture-periodicity, and Jensen-Shannon Divergence, which is considered to be a smoothed and symmetrized version of the Kullback-Leibler Divergence, have been effectively used for the development of the automated defect detection scheme for patterned textures. Experiments on real fabric images belonging to 3 major wallpaper groups with defects show that the proposed method is robust in finding fabric defects. The absence of a training stage with defect-free samples for decision-boundaries or thresholds, the unsupervised method of identifying defects using cluster analysis, and high success rates are the novelties of this proposed method. Thus, the proposed method can contribute to the development of computerized defect detection in fabric industries.

\section{REFERENCES}

[1] H.S.M. Coxeter and W.O.J. Moser, Generators and Relations for Discrete Groups, $4^{\text {th }}$ ed., SpringerVerlag, New York, 1980.

[2] D. Schattschneider, D., "The Plane Symmetry Groups: Their Recognition and Notation," Am. Math. Monthly, Vol.85, No.6, 1978, pp.439-450.

[3] H.Y.T. Ngan and G.H.K. Pang, "Regularity Analysis for Patterned Texture Inspection," IEEE Trans. on Autom. Sci. and Eng., Vol.6, No.1, 2009, pp.131-144.

[4] H.Y.T. Ngan, G.H.K. Pang and N.H.C. Yung, "Performance Evaluation for Motif-Based Patterned Texture Defect Detection," IEEE Trans. on Autom. Sci. and Eng., Vol.7, No.1, 2010, pp.58-72.

[5] H.Y.T. Ngan, G.K.H. Pang, S.P. Yung and M.K. Ng, "Wavelet based methods on patterned fabric defect detection,” Pattern Recognit., Vol.38, No.4, 2005, pp.559-576.

[6] H.Y.T. Ngan and G.H.K. Pang, "Novel method for patterned fabric inspection using Bollinger bands, ” Opt. Eng., Vol.45, No.8, 2006, pp.087202-1-15.

[7] F. Tajeripour, E. Kabir and A. Sheikhi, "Fabric Defect Detection Using Modified Local Binary Patterns," Proc. of the Int. Conf. on Comput. Intel. and Multimed. Appl., Sivakasi, Tamilnadu, India, December, 2007, pp.261-267.

[8] H.Y.T. Ngan, G.H.K. Pang and N.H.C. Yung, "Motif-based defect detection for patterned fabric," Pattern Recognit., Vol.41, No.6, 2008, pp.1878-1894.

[9] H.Y.T. Ngan and G.H.K. Pang, "Ellipsoidal decision regions for motif-based patterned fabric defect detection," Pattern Recognit., Vol.43, No.6, 2010, pp.2132-2144.

[10] R.T. Chin and C.A. Harlow, "Automated visual inspection: A survey," IEEE Trans. on Pattern Anal. and Mach. Intel., Vol.4, No.6, 1982, pp.557-573.

[11] B.H. Khalaj and T. Kailath, "Patterned wafer inspection by high resolution spectral estimation tech- 
niques," Mach. Vision and Appl., Vol.7, 1994, pp.178-185.

[12] P. Xie and S.U. Guan, "A golden-template self-generating method for patterned wafer inspection," Mach. Vision and Appl., Vol.12, 2000, pp.149-156.

[13] D.M. Endres, J.E. Schindelin, “A New Metric for Probability Distributions," IEEE Trans. on Info. Theory, Vol.49, No.7, 2003, pp.1858-1860.

[14] S. Theodoridis and K. Koutroumbas, Pattern Recognition, $4^{\text {th }}$ ed., Academic Press, CA, 2009.

[15] V. Asha, N.U. Bhajantri, P. Nagabhushan, "Automatic Detection of Texture Defects using TexturePeriodicity and Gabor Wavelets," in: Venugopal, K.R., and Patnaik, L.M. (eds.): ICIP 2011, Communication and Computer Information Series (CCIS), Vol.157, Springer-Verlag, Berlin Heidelberg, 2011, pp.548-553.

[16] R.C. Gonzalez and R.E. Woods, Digital Image Processing, Pearson Prentice Hall, New Delhi, 2008.

[17] T. Fawcett, "An introduction to ROC analysis," Pattern Recognit. Lett., Vol.27, No.8, 2006, pp.861874.

[18] C.D. Brown, H.T. Davis, "Receiver operating characteristics curves and related decision measures: A tutorial," Chemom. and Intell. Lab. Syst., Vol.80, No.1, 2006, pp.24-38.

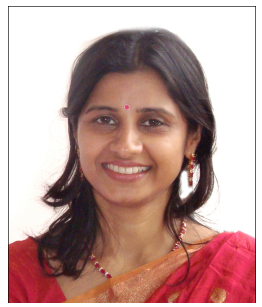

\section{Asha}

She received her Bachelor and Master degrees in Computer Science from Mysore University during 1991 and 1993 respectively. Currently, she is working as an Assistant Professor in the Department of Computer Applications at the New Horizon College of Engineering in Bangalore. Her research area includes image processing and pattern recognition.

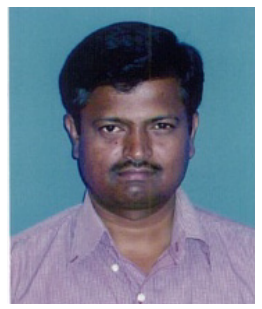

\section{Dr. N.U. Bhajantri}

He received his M.Tech and Ph.D from the Indian Institute of Technology Delhi and his Ph.D from Mysore University in 1999 and 2007 respectively. Currently, he is a Professor at the Govt. College of Engineering, Chamarajanagar, in the Mysore District of Karnataka, India. He works in the broad area of image processing, pattern recognition, and other related fields.

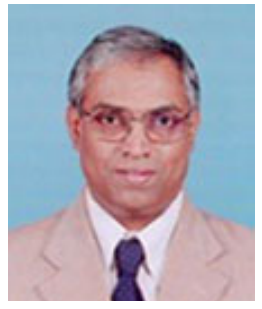

\section{Dr. P. Nagabhushan}

He received his M.Tech and Ph.D from Birla Institute of Technology, Mesra, Ranchi, and Mysore University during 1983 and 1988 respectively. Currently, he is in the Department of Computers and Studies at the University of Mysore in Mysore, India. He works in the broad area of cognition-recognition, pattern recognition, digital image processing, object recognition, data and knowledge mining, document image and other related fields. 